\title{
Efficient steady-state analysis of Second-Order Fluid Stochastic Petri Nets
}

\author{
M. Gribaudo and R. Gaeta* \\ Dipartimento di Informatica, Università di Torino, Corso Svizzera 185, 10149, \\ Torino, Italy
}

\begin{abstract}
This paper presents an efficient solution technique for the steady-state analysis of the second-order Stochastic Fluid Model underlying a second-order Fluid Stochastic Petri Net (FSPN) with constant flow and transition rates, and a single fluid place. The new solution technique is an extension of existing solution techniques developed for (first-order) Fluid Models; the solution algorithm uses upwind semidiscretization and Matrix Geometric techniques to efficiently compute the steadystate joint probability of the discrete states and the fluid level. The effectiveness of our technique is proven first on a simple producer-consumer second-order FSPN model and then on a complex example taken from the literature where the analysis of the completion time distribution and the packet loss probability of short-lived TCP connections is investigated through the decomposition of a model for the whole system into several (simpler) sub-models; the interaction among the different sub-models is handled by iterating their solution until the complete model solution converges according to a fixed point algorithm. The introduction of a second-order FSPN in a fixed point iteration scheme has been made possible thanks to the efficiency of the proposed solution technique.
\end{abstract}

Key words: second order stochastic fluid model, fluid stochastic Petri nets, matrix geometric, TCP

\section{Introduction}

Stochastic fluid models are a class of analytic models that have recently drawn the attention of many researchers for the performance evaluation of complex

* Corresponding Author. Phone: +39 01167067 70; Fax: +39 011751603

Email address: marcog@di.unito.it,rossano@di.unito.it (M. Gribaudo and R. Gaeta). 
computer and communication systems.

In the simplest first-order fluid models the fluid flow rate is constant with a given mean value. A first example of first-order fluid based approaches is the one in which queues and buffers are approximated by fluid. In the work done by D. Mitra in [4], a producer-consumer model, with a fixed number of producers and a single consumer, with an intermediate fluid buffer is presented. In the proposed model, a Markov chain describes the state of the sources (i.e. it counts how many producers are active). In each state an input rate is computed based on the number of active producers. The difference between this value and the output rate of the consumer gives an instantaneous rate of change of the fluid buffer. A system of first-order differential equation is then written to completely characterize the model. Efficient transient analysis of first-order fluid models with constant rates is proposed in [21].

The simple first-order model of [4] is extended to consider fluid-dependent flow rates in [9] where different classes of sources, finite buffer, and production rates dependent on buffer occupancy are considered. This class of models is used to describe a rate-based congestion control of a high speed network with loss priorities.

Second-order fluid models, on the other hand, are models where the fluid flow is not considered constant in a given state, but it is defined by a mean flow rate and a variance. These models are referred to as second-order models, since they require the solution of a system of second-order differential equations. Usually these models describe the whole system using only continuous quantities. Discrete states are not considered and discrete quantities are approximated by continuous ones. The theory behind these approaches is based on the central limit theorem: a sum of a large number of random variables can be approximated by a Normal distribution characterized by a mean that corresponds to the sum of the means of the various random variables, and by a variance that corresponds to the sum of the various variances. The normal distribution can then be approximated by the solution of a diffusion process that is expressed through a second-order differential equation. Models are expressed as standard queuing networks with general service time distributions. Only the first two moments of these distributions (i.e. their mean and their variance) are required to find the parameters for the diffusion equation. This approach has been extended in order to consider finite capacity queues and complex server policies. It has been used to study several applicative problems, such as cellular telephone cells or ABR traffic sources. A good reference to these kinds of models can be found in [8]. Further references to works using second-order stochastic fluid models are $[17,3]$.

In [6] a general solution technique for the transient analysis of second-order stochastic fluid models with fluid-dependent flow rates is presented. 
Stochastic fluid models are useful analytic tools for the performance analysis of complex systems but despite their mathematical elegance they can be hard to solve, since they require the direct manipulation of Markov chains and (sometimes complex) differential equations. For this reason, higher level mathematical formalisms have been introduced in the literature for the specification of such models. For first-order fluid models, an example of such higher-level formalisms is first-order Fluid Stochastic Petri Nets (FSPN) [22,15,7,2,14]; FSPN models are analyzed by writing the equations of the underlying stochastic process (a first-order fluid model) and then by solving them numerically. The equations are written by decoupling the discrete part of the model from the continuous one. First, the set of possible discrete states (the discrete state space) is generated using conventional Petri net techniques; then a system of partial differential equations is generated. This system includes one equation for each discrete state that the system may reach, and a partial derivative for each fluid place, plus a partial derivative for the time. Even though equations can be written for every FSPN, these equations are so hard to solve that, except for special classes of FSPN models [13,12], only models with one or two fluid places may be analyzed.

For second-order fluid models, second-order FSPN have been introduced in [23]. The most interesting feature of second-order FSPN (and all their extensions $[24,25]$ ) is that equations can be written, and thus analytical and numerical solution techniques can be applied. The only difference with respect to the FSPN solution techniques is that second-order partial differential equations must be solved in this framework. Numerical solution techniques for second-order PDEs have almost the same complexity as the first-order solution techniques.

The contribution of this paper is two-fold:

- we present an efficient solution technique for the steady-state analysis of the second-order stochastic fluid model underlying a second-order FSPN with constant flow and transition rates and variance, and a single fluid place. The new solution technique is an extension of existing solution techniques developed for first-order fluid models; the effectiveness of our technique is proven first on a simple producer-consumer second-order FSPN model.

In the current work we restrict our attention to second-order stochastic fluid models with constant rates that represent a sub-class of the general second-order stochastic fluid models considered in [6]. Furthermore, we develop a solution technique for the steady-state analysis of this model subclass while the work in [6] focuses on the transient analysis.

- not many applications of second-order FSPN can be found in the literature [26]. We develop a second-order FSPN model for a complex example taken from the literature where the analysis of the completion time distribution and the packet loss probability of short-lived TCP connections 
is investigated through the decomposition of a model for the whole system into several (simpler) sub-models; the interaction among the different sub-models is handled by iterating their solution until the complete model solution converges according a fixed point algorithm. The introduction of a second-order FSPN in a fixed point iteration scheme has been made feasible thanks to the efficiency of the proposed solution technique.

The outline of the paper is as follows: Sections 2 and 3 contain brief introductions to second-order fluid models and second-order FSPNs, respectively. Section 4 describes the solution technique for second-order fluid models we developed, Section 5 describes the second-order FSPN model we developed to analyze the performance of short-lived TCP connections, and Section 6 draws some conclusions and outlines future extensions of this work.

\section{Second-order stochastic fluid models}

A second-order stochastic fluid model is a stochastic process defined on a mixed discrete-continuous state space. In particular, we follow the definition proposed in [6], and we define it as a stochastic process $\mathcal{M}(\tau)=\{M(\tau), X(\tau)\}$. $M(\tau) \in \mathcal{S}$ represents the state of the modulating Markov chain (whose state space is defined by set $\mathcal{S}) . X(\tau) \in[0, \infty)$ represent the accumulated fluid at time $\tau$, and it is a non-negative real number. In this paper we will consider only bounded models, that is, models where the fluid level is bounded by real number $X(\tau) \in[0, B]$ The infinitesimal generator of the underlying Markov chain it is represented by a constant Matrix $\boldsymbol{Q}$. In particular $q_{i j}$ denotes the transition rates from state $i$ to state $j$, if $i \neq j$, and $q_{i i}=-\sum_{j \neq i} q_{i j}$. According to the Brownian theory, $X(\tau+h)-X(\tau)$ in the $X(\tau)$ process over $[t, t+h]$ under a given environmental state $k$ is assumed to be normally distributed with mean $\mu_{k}$ and variance $\sigma_{k}^{2}$. The two parameters can be defined as:

$$
\lim _{h \rightarrow 0} \frac{E[X(\tau+h)-X(\tau) \mid M(\tau)=k]}{h}=\mu_{k},
$$

and

$$
\lim _{h \rightarrow 0} \frac{\operatorname{Var}[X(\tau+h)-X(\tau) \mid M(\tau)=k]}{h}=\sigma_{k}^{2} .
$$

Note that, with respect to [6], in this case we do not allow parameters $\mu_{k}$ and $\sigma_{k}^{2}$ to be dependent on the fluid level.

Let us define the probability density function $\pi(k, x, \tau)$ as the probability of being in state $k$ at time $\tau$ with fluid level $x$, that is:

$$
\pi(k, x, \tau)=\lim _{\Delta x} \frac{\operatorname{Pr}\{x<X(\tau) \leq x+\Delta x, M(\tau)=k\}}{\Delta x} .
$$


In the following we will express this probability $\boldsymbol{\pi}(x, \tau)=\{\pi(k, x, \tau), k \in \mathcal{S}\}$ in vector form. If we define $\boldsymbol{R}=\operatorname{diag}\left(\mu_{k}, k \in \mathcal{S}\right)$, and $\boldsymbol{V}=\operatorname{diag}\left(\sigma_{k}^{2}, k \in \mathcal{S}\right)$, then the governing equation is:

$$
\frac{\partial \boldsymbol{\pi}(x, \tau)}{\partial \tau}+\frac{\partial \boldsymbol{\pi}(x, \tau) \boldsymbol{R}}{\partial x}=\frac{1}{2} \frac{\partial^{2} \boldsymbol{\pi}(x, \tau) \boldsymbol{V}}{\partial x^{2}}+\boldsymbol{\pi}(x, \tau) \boldsymbol{Q}
$$

Following [24], if the system has steady state, then the steady state version of equation 1 is:

$$
\frac{d \boldsymbol{\pi}(x)}{d x} \boldsymbol{R}-\frac{1}{2} \frac{d^{2} \boldsymbol{\pi}(x)}{d x^{2}} \boldsymbol{V}=\boldsymbol{\pi}(x) \boldsymbol{Q}
$$

\subsection{Boundary conditions}

When dealing with second-order fluid models, boundary plays an important role in describing the model behavior. Boundaries are considered as barriers that prevent the fluid from going beyond them. In particular three different cases may arise depending on the variance and on the sign of the mean flow rate. When the variance is different from zero, we have a reflecting state. No probability mass may arise at these kind of boundaries. If the variance is equal to zero, then we may have absorbing states if the mean flow is directed through the barrier (i.e. negative for the lower bound and positive for the upper bound), and emitting states if the rate is opposed to the barrier. Boundary conditions are quite complex to treat analytically: for a complete analysis the reader should refer to [6]. In particular, when considering absorbing states, probability mass builds up at the boundary. In the following we will call $c(k, b, \tau)$ the probability mass at boundary $b$ (either $b=0$ or $b=B$ ). It can be proven that for emitting and reflecting states $k, c(k, b, \tau)=0$ (see [6]). Note that

$$
c(k, b, \tau)=\lim _{h \rightarrow b} \int_{h}^{b} \pi(k, x, \tau) d x .
$$

It can also be shown that boundary conditions for the various types of states in steady state can be mixed into a single matrix equation, which is for the lower boundary:

$$
\boldsymbol{\pi}(0) \boldsymbol{R}-\left.\frac{1}{2} \frac{\partial \boldsymbol{\pi}(x)}{d x} \boldsymbol{V}\right|_{x=0}=\boldsymbol{c}(0) \boldsymbol{Q}
$$


and for the upper boundary:

$$
-\boldsymbol{\pi}(B) \boldsymbol{R}+\left.\frac{1}{2} \frac{\partial \boldsymbol{\pi}(x)}{d x} \boldsymbol{V}\right|_{x=B}=\boldsymbol{c}(B) \boldsymbol{Q} .
$$

\section{Second-order Fluid Stochastic Petri Nets}

Fluid Stochastic Petri Nets (FSPN) were introduced by K. S. Trivedi and V. G. Kulkarni in [22], and then extended in [15]. A FSPN is an extension of stochastic Petri net in which continuous quantities may be included directly in the model. A FSPN has two types of places: discrete places (containing a non-negative number of tokens) and continuous places (containing fluid). Discrete places are drawn as single circle, while fluid places are represented by two concentric circles. Transition firings are determined by both discrete and continuous places, and fluid flow is permitted through enabled timed transitions. Transitions may be either timed, when they have associated an exponential firing time, or immediate, when they fire in zero time. Timed transitions are drawn with boxes, while immediate transitions are represented by bars. To each transition a guard may be associated. A guard is boolean function of the state space $\mathcal{G}:(\mathcal{T} \times \mathcal{S}) \rightarrow\{0,1\}$ where $\mathcal{T}$ represents the set of transitions and $\mathcal{S}$ the discrete-continuous state space. A transition $t$ may fire in state $\mathcal{M}$ only if $\mathcal{G}(t, \mathcal{M})=1$. Guards associated with timed transitions may depend upon the marking of both the discrete and the continuous places, while guards associated with immediate transitions may depend only upon the discrete part of the marking. Fluid flow is determined by fluid arcs that connect timed transitions to fluid places. Each fluid arc that goes from transition $t$ to fluid place $c$ has associated a (possibly state $\mathcal{M}$ dependent) flow rate $r((t, c), \mathcal{M})$. If transition $t$ is enabled in state $\mathcal{M}$, then fluid flows across the arc at $r((t, c), \mathcal{M})$ fluid units per time units. If the arc is directed from the transition to the place, then the fluid enters the fluid place. If the arc is directed from the place to the transition, fluid flows out of the place. Standard arcs are represented by standard arrows, while fluid arcs are represented as a double line, to suggest a pipe. Each fluid place $c$ may have an upper boundary $B_{c}$ and has an implicit lower boundary at level 0 . When a boundary level is reached, fluid flow is stopped to maintain the constraint.

Second-order fluid stochastic Petri nets have been introduced in [23]. Those models are an extension of the FSPN formalism. In second-order FSPN, fluid does not flow at a constant rate, but at randomly variable rate. The flow rate is specified by a mean flow rate and a flow variance. These kind of models are called second-order models since their solution involves the solution of a system of second-order partial differential equations. In second-order FSPN, each fluid arc has associated a mean flow rate and a variance. Then the potential flow 
rate in each discrete state is computed as the sum of the flow rate of the input arcs that connect enabled timed transitions to that place, minus the sum of the flow rate of the output arcs that connect that place to enabled timed transitions. The potential variance of the state is computed as the sum of the variance of all the fluid arcs that connect that place to enabled timed transitions, regardless of the direction of the arc. Figure 1 represents all the graphic primitives of the second-order FSPN used in this paper. Note that, similarly to [2], we have chosen to use fluid transitions instead of fluid arcs. In this case, the flow rates and flow variances are associated to transitions rather than to fluid arcs. Fluid transitions can only be connected to discrete places using test and inhibitor arcs. Fluid arcs are not labeled and their purpose is just to connect fluid transitions to fluid places.

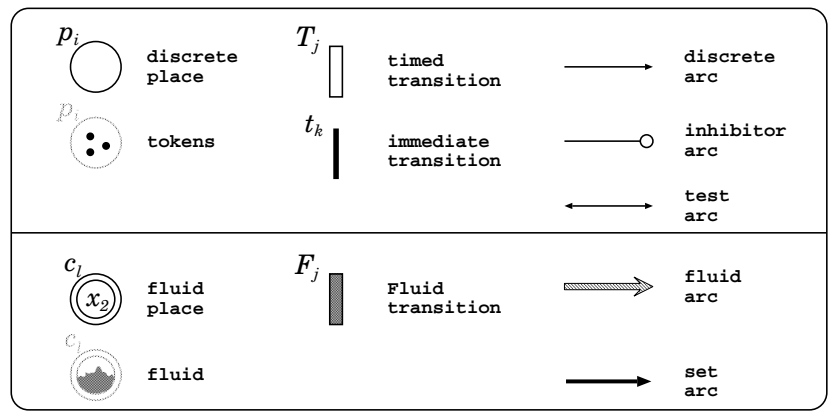

Fig. 1. Second-order FSPN primitives

Automatic techniques exist to compute second-order stochastic fluid model underlying a second-order FSPN, such as the one presented in [24].

Some extension of second-order FSPN have been presented in the literature. For example, the formalism presented in [24] includes fluid jumps, and the one proposed in [25] (called Non-Markovian second-order FSPN) makes it possible to also use timed transitions with a non exponential firing time, as long as only one general transition is enabled in a discrete marking

\section{The proposed solution technique}

The steady-state solution of the proposed second-order FSPN model has been computed by using an extension of the solution technique proposed in [16]. The solution algorithm uses upwind semi-discretization and matrix geometric techniques to efficiently compute the steady state joint probability of the discrete states and the fluid level.

Consider a second-order FSPN model with a single bounded fluid place, in which neither the flow rates associated to fluid arcs nor the firing rates associated to timed transition, nor the fluid flow variance depend on the continuous 
part of the net. The equation that describes the steady state solution $\boldsymbol{\pi}(x)$ is

$$
\frac{d \boldsymbol{\pi}(x)}{d x} \boldsymbol{R}-\frac{1}{2} \frac{d^{2} \boldsymbol{\pi}(x)}{d x^{2}} \boldsymbol{V}=\boldsymbol{\pi}(x) \boldsymbol{Q}
$$

where $\boldsymbol{R}$ is a diagonal matrix whose element represents the mean flow rate in every state, $\boldsymbol{V}$ is a diagonal matrix whose element contains the variance of the fluid flow in every state, and $\boldsymbol{Q}$ is the transition matrix of the discrete part of the model.

Numerical solution of (6) can be obtained using upwind discretization [15] when the variance in that state is 0 , and with standard second-order discretization when the variance is greater than 0 . In the upwind discretization, the derivative along the fluid level $x$ is approximated by the "upwind" finite difference. The fluid level is discretized with step-size $\Delta x$. Let us use the notation $\boldsymbol{\pi}^{(i)}=\boldsymbol{\pi}(i \Delta x)$. If $r_{j}>0$ (the actual flow rate in discrete state $j$, where state $j$ means : $j$ sources are active), then

$$
\frac{d \pi_{j}(i \Delta x)}{d x} \approx \frac{\pi_{j}^{(i)}-\pi_{j}^{(i-1)}}{\Delta x} .
$$

Instead, if $r_{j}<0$, the derivative is approximated by

$$
\frac{d \pi_{j}(i \Delta x)}{d x} \approx \frac{\pi_{j}^{(i+1)}-\pi_{j}^{(i)}}{\Delta x} .
$$

When the variance is different from 0 , then we approximate the first-order derivative with

$$
\frac{d \pi_{j}(i \Delta x)}{d x} \approx \frac{\pi_{j}^{(i+1)}-\pi_{j}^{(i-1)}}{2 \Delta x} .
$$

and the second-order derivative with:

$$
\frac{d^{2} \pi_{j}(i \Delta x)}{d x^{2}} \approx \frac{\pi_{j}^{(i+1)}-2 \pi_{j}^{(i)}+\pi_{j}^{(i-1)}}{\Delta x^{2}} .
$$

This way the discretized version of (6) becomes

$$
\begin{gathered}
\frac{\boldsymbol{\pi}^{(i)}-\boldsymbol{\pi}^{(i-1)}}{\Delta x} \boldsymbol{R}^{+}+\frac{\boldsymbol{\pi}^{(i+1)}-\boldsymbol{\pi}^{(i)}}{\Delta x} \boldsymbol{R}^{-}+ \\
\frac{\boldsymbol{\pi}^{(i+1)}-\boldsymbol{\pi}^{(i-1)}}{2 \Delta x} \boldsymbol{R}^{(n z)}-\frac{\pi_{j}^{(i+1)}-2 \pi_{j}^{(i)}+\pi_{j}^{(i-1)}}{2 \Delta x^{2}} \boldsymbol{V}=\boldsymbol{\pi}^{(i)} \boldsymbol{Q}
\end{gathered}
$$

where the diagonal matrices $\boldsymbol{R}^{+}, \boldsymbol{R}^{-}, \boldsymbol{R}^{(n z)}$ are defined as 


$$
\begin{aligned}
\boldsymbol{r}_{i i}^{+} & = \begin{cases}r_{i i}, & \text { if } r_{i i}>0 \wedge v_{i i}=0, \\
0, & \text { otherwise }\end{cases} \\
\boldsymbol{r}_{i i}^{-} & = \begin{cases}r_{i i}, & \text { if } r_{i i}<0 \wedge v_{i i}=0, \\
0, & \text { otherwise }\end{cases} \\
\boldsymbol{r}_{i i}^{(n z)} & = \begin{cases}r_{i i}, & \text { if } v_{i i} \neq 0, \\
0, & \text { otherwise }\end{cases}
\end{aligned}
$$

Using the notation

$$
\begin{aligned}
& \boldsymbol{X}^{+}=\frac{1}{\Delta x}\left(\boldsymbol{R}^{+}+\frac{1}{2} \boldsymbol{R}^{(n z)}\right)+\frac{1}{2} \frac{1}{\Delta x^{2}} \boldsymbol{V}, \\
& \boldsymbol{X}^{-}=-\frac{1}{\Delta x}\left(\boldsymbol{R}^{-}+\frac{1}{2} \boldsymbol{R}^{(n z)}\right)+\frac{1}{2} \frac{1}{\Delta x^{2}} \boldsymbol{V},
\end{aligned}
$$

(7) can be rewritten as

$$
-\boldsymbol{\pi}^{(i-1)} \boldsymbol{X}^{+}+\boldsymbol{\pi}^{(i)}\left[\boldsymbol{X}^{+}+\boldsymbol{X}^{-}\right]-\boldsymbol{\pi}^{(i+1)} \boldsymbol{X}^{-}=\boldsymbol{\pi}^{(i)} \boldsymbol{Q},
$$

or, setting $\boldsymbol{C}=\boldsymbol{Q}-\boldsymbol{X}^{+}-\boldsymbol{X}^{-}$:

$$
\boldsymbol{\pi}^{(i-1)} \boldsymbol{X}^{+}+\boldsymbol{\pi}^{(i)} \boldsymbol{C}+\boldsymbol{\pi}^{(i+1)} \boldsymbol{X}^{-}=\mathbf{0} .
$$

\subsection{Boundary conditions}

In order to discretize the boundary conditions, we start by discretizing Equation 3 , from which we obtain (with $b=0$ and $b=B$ ):

$$
\boldsymbol{c}(b)=\boldsymbol{\pi}(b) \Delta x .
$$

In the following, we call $\boldsymbol{\pi}^{(0)}$ the discretized version of the lower boundary $\boldsymbol{\pi}^{(0)}$, and $\boldsymbol{\pi}^{(m)}$ the discretized version of the upper boundary $\boldsymbol{\pi}^{(B)}$. Since we chosen to use an upwind discretization scheme, the upwind direction must be used when discretizing $\boldsymbol{\pi}(b)$. In particular we have to discretize it in three different ways, depending on direction of the flow, and on the value of the variance. Table 1 shows the various choices that must be made for both the upper and lower boundaries. Note that when the variance is zero, $\boldsymbol{\pi}(b)$ is chosen to be

the value at the boundary if the flow is directed out of the boundary, to be 
Table 1

Discretization of $\boldsymbol{\pi}(b)$.

\begin{tabular}{|l|c|c|}
\hline & Lower Bound & Upper bound \\
\hline \hline$r_{i i}>0 \wedge v_{i i}=0$ & $\boldsymbol{\pi}^{(0)}$ & $\boldsymbol{\pi}^{(m-1)}$ \\
\hline$r_{i i}<0 \wedge v_{i i}=0$ & $\boldsymbol{\pi}^{(1)}$ & $\boldsymbol{\pi}^{(m)}$ \\
\hline$v_{i i} \neq 0$ & $\frac{\boldsymbol{\pi}^{(0)}+\boldsymbol{\pi}^{(1)}}{2}$ & $\frac{\boldsymbol{\pi}^{(m-1)}+\boldsymbol{\pi}^{(m)}}{2}$ \\
\hline \hline
\end{tabular}

its near value if the flow is directed into the boundary, and to be the average of the two if the variance is different from zero. Using Table 1 and inserting Equation 11 into Equations 4 and 5 we obtain:

$$
\begin{gathered}
\frac{\boldsymbol{\pi}^{(0)}}{\Delta x} \boldsymbol{R}^{+}+\frac{\boldsymbol{\pi}^{(1)}}{\Delta x} \boldsymbol{R}^{-}+ \\
\frac{\boldsymbol{\pi}^{(0)}+\boldsymbol{\pi}^{(1)}}{2 \Delta x} \boldsymbol{R}^{(n z)}-\frac{\boldsymbol{\pi}^{(1)}-\boldsymbol{\pi}^{(0)}}{2 \Delta x^{2}} \boldsymbol{V}=\boldsymbol{\pi}^{0} \boldsymbol{Q}, \\
-\frac{\boldsymbol{\pi}^{(m-1)}}{\Delta x} \boldsymbol{R}^{+}-\frac{\boldsymbol{\pi}^{(m)}}{\Delta x} \boldsymbol{R}^{-}+ \\
-\frac{\boldsymbol{\pi}^{(m-1)}+\boldsymbol{\pi}^{(m)}}{2 \Delta x} \boldsymbol{R}^{(n z)}+\frac{\boldsymbol{\pi}^{(m)}-\boldsymbol{\pi}^{(m-1)}}{2 \Delta x^{2}} \boldsymbol{V}=\boldsymbol{\pi}^{m} \boldsymbol{Q},
\end{gathered}
$$

If we use the notation in Equation 8, and define $\boldsymbol{C}^{+}=\boldsymbol{Q}-\boldsymbol{X}^{+}$and $\boldsymbol{C}^{-}=$ $\boldsymbol{Q}-\boldsymbol{X}^{-}$, the boundary conditions become:

$$
\begin{aligned}
\boldsymbol{\pi}^{(0)} \boldsymbol{C}^{+}+\boldsymbol{\pi}^{(1)} \boldsymbol{X}^{-} & =\mathbf{0} \\
\boldsymbol{\pi}^{(m-1)} \boldsymbol{X}^{+}+\boldsymbol{\pi}^{(m)} \boldsymbol{C}^{-} & =\mathbf{0}
\end{aligned}
$$

The discretization scheme is stable only if $\left|\boldsymbol{X}^{+}\right|>0$ and $\left|\boldsymbol{X}^{-}\right|>0$. This is true if:

$$
\forall i \mid \sigma_{i}^{2}>0: \frac{\sigma_{i}^{2}}{\Delta x^{2}}>\frac{\left|\mu_{i}\right|}{\Delta x}
$$

that is only if $\Delta x<\min _{i \mid \sigma_{i}^{2}>0 \wedge \mu_{i} \neq 0}\left(\frac{\sigma_{i}^{2}}{\left|\mu_{i}\right|}\right)$. As we will see, this is not a problem for the solution algorithm that will be presented into the next section, since it is almost independent on the choice of $\Delta x$. 


\subsection{Solution of the discretized equations}

Equation 10 has a particular tridiagonal structure, as shown in Figure 2, and can be solved using matrix geometric techniques such as the one proposed in [18], following the steps presented in [16]. In particular, denoting by $\Lambda$ and $\Gamma$

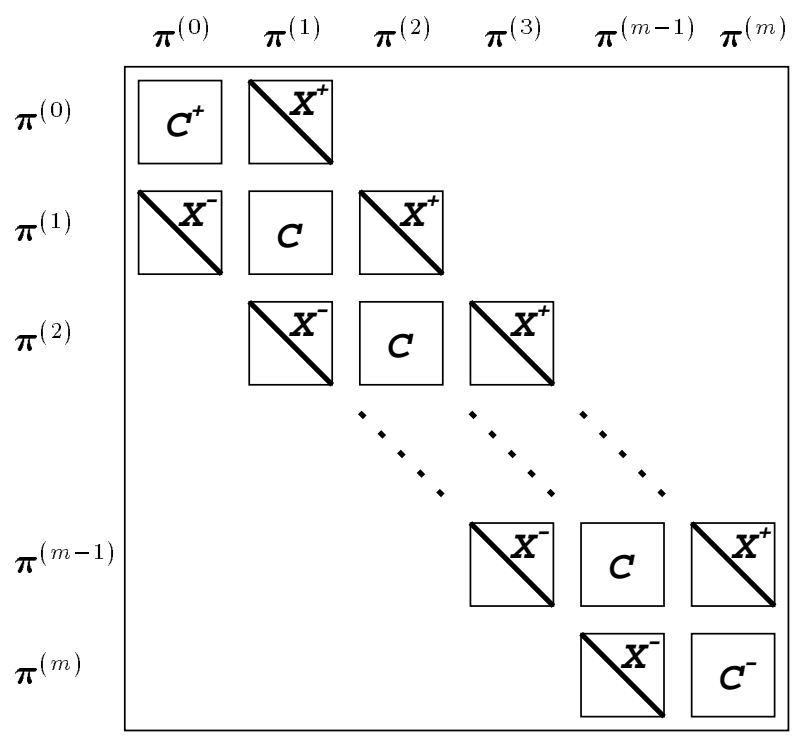

Fig. 2. Coefficient matrix of the discretized system of equations

the solution of the two matrix equations:

$$
\begin{aligned}
\boldsymbol{X}^{+}+\Lambda \boldsymbol{C}+\boldsymbol{\Lambda}^{2} \boldsymbol{X}^{-} & =0 \\
\boldsymbol{X}^{-}+\boldsymbol{\Gamma} \boldsymbol{C}+\boldsymbol{\Gamma}^{2} \boldsymbol{X}^{+} & =0
\end{aligned}
$$

the solution of the original system of equations is obtained by

$$
\boldsymbol{\pi}^{(i)}=\boldsymbol{u}_{\mathbf{0}} \boldsymbol{\Lambda}^{i}+\boldsymbol{u}_{\boldsymbol{m}} \boldsymbol{\Gamma}^{m-i}
$$

$\boldsymbol{u}_{\mathbf{0}}$ and $\boldsymbol{u}_{\boldsymbol{m}}$ can be computed by solving the linear system:

$$
\left|\begin{array}{ll}
\boldsymbol{u}_{\mathbf{0}} & \boldsymbol{u}_{\boldsymbol{m}}
\end{array}\right| \boldsymbol{J}=0
$$

where the coefficient matrix $\boldsymbol{J}$ is defined by:

$$
\boldsymbol{J}=\left|\begin{array}{cc}
\boldsymbol{C}^{+}+\boldsymbol{\Lambda} \boldsymbol{X}^{-} & \boldsymbol{\Lambda}^{m-1} \boldsymbol{X}^{+}+\boldsymbol{\Lambda}^{m} \boldsymbol{C}^{-} \\
\boldsymbol{\Gamma}^{m-1} \boldsymbol{X}^{-}+\boldsymbol{\Gamma}^{m} \boldsymbol{C}^{+} & \boldsymbol{C}^{-}+\boldsymbol{\Gamma} \boldsymbol{X}^{+}
\end{array}\right|
$$


under the normalization condition

$$
\left[\sum_{i=0}^{\infty} \boldsymbol{\pi}^{(i)}\right] \mathbf{1}=\left[\boldsymbol{u}_{\mathbf{0}} \sum_{i=0}^{m} \Lambda^{i}+\boldsymbol{u}_{\boldsymbol{m}} \sum_{i=0}^{m} \Gamma^{i}\right] \mathbf{1}=1 .
$$

Matrix $\Lambda$ and $\Gamma$ can be obtained in a very efficient manner using the technique of Latouche and Ramaswami [18]. They can be determined by the iterative procedure called "logarithmic reduction", that has logarithmic convergence. In this case, the logarithmic reduction algorithm must be performed two times in order to compute both $\Lambda$ and $\Gamma$. The computation of $\Lambda^{m}, \Gamma^{m}, \sum_{i=0}^{m} \Lambda^{i}$ and $\sum_{i=0}^{m} \Gamma^{i}$ can also be carried out in logarithmic time.

\subsection{A simple example}

In order to illustrate the power of the proposed technique, we will compare it to other standard discretization techniques for the solution of a simple producerconsumer example.

Figure 3 presents a simple second-order FSPN model of a fluid buffer, where $N$ producers and $K$ consumers store their jobs. The buffer is represented by fluid place $c_{0}$, and it is bounded to a maximum fluid level $B_{0}$. Places $P_{0}$ and $P_{1}$ represent the pool of $N$ producers $\left(P_{0}\right.$ the ones that are inactive, and $P_{1}$ the one that are actually producing), and places $P_{2}$ and $P_{3}$ the pool of $K$ consumer ( $P_{3}$ the idle ones, and $P_{2}$ the consuming ones). Producers (resp. consumers) can go stop producing (consuming) by the firing of transition $T_{1}\left(T_{2}\right)$, and may resume their work by the firing of transition $T_{0}\left(T_{3}\right)$. The production is represented by fluid transition $F_{0}$, with mean rate $\mu_{0}$ and variance $\sigma_{0}^{2}$, and the consumption by fluid transition $F_{1}$, with parameters $\mu_{1}$ and $\sigma_{1}^{2}$. All the transitions (both fluid and discrete) have infinite server semantics: their actual rate is the product of their parameters by their enabling degree.

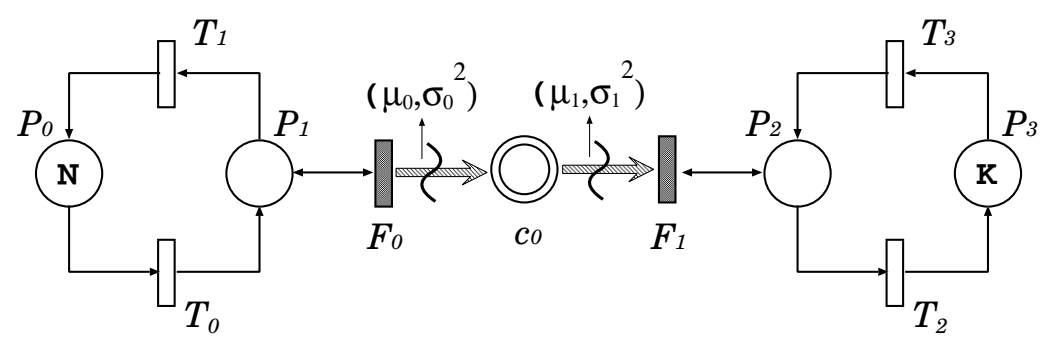

Fig. 3. A simple second-order FSPN producer-consumer model

In all the presented example, the parameters were set as follows (the rate of transition $T_{i}$ is denoted as $\lambda_{i}$ ): 
Table 2

CPU times for fixed number of producers and consumer for decreasing $\Delta x$.

\begin{tabular}{|r|r|r|r|r|}
\hline \hline$B_{0}$ & $\Delta X$ & $m+1$ & standard & new \\
\hline \hline 1.0 & 0.1 & 10 & $23 \mathrm{~s}$. & $<1 \mathrm{s.}$ \\
\hline 1.0 & 0.05 & 20 & $74 \mathrm{~s}$. & $1 \mathrm{~s}$. \\
\hline 1.0 & 0.02 & 50 & $165 \mathrm{~s}$. & $6 \mathrm{~s}$. \\
\hline 1.0 & 0.01 & 100 & $355 \mathrm{~s}$. & $6 \mathrm{~s}$. \\
\hline 1.0 & 0.001 & 1000 & n.c. s. & $6 \mathrm{~s}$. \\
\hline 1.0 & 0.0001 & 10000 & n.c. s. & $7 \mathrm{~s}$. \\
\hline \hline
\end{tabular}

Table 3

CPU times for fixed number of producers and consumer for increasing $B_{0}$.

\begin{tabular}{|r|r|r|r|r|}
\hline \hline$B_{0}$ & $\Delta X$ & $m+1$ & standard & new \\
\hline \hline 0.2 & 0.1 & 2 & $247 \mathrm{~s}$. & $73 \mathrm{s.}$ \\
\hline 0.5 & 0.1 & 5 & $485 \mathrm{~s}$. & $69 \mathrm{s.}$ \\
\hline 1.0 & 0.1 & 10 & $486 \mathrm{~s}$. & $72 \mathrm{s.}$ \\
\hline 10.0 & 0.1 & 100 & $4,485 \mathrm{~s}$. & $257 \mathrm{~s}$. \\
\hline 100.0 & 0.1 & 1000 & n.c. & $269 \mathrm{s.}$ \\
\hline 1000.0 & 0.1 & 10000 & n.c. & $296 \mathrm{~s}$. \\
\hline 10000.0 & 0.1 & 100000 & n.c. & $2,392 \mathrm{~s}$. \\
\hline \hline
\end{tabular}

$$
\begin{gathered}
\lambda_{0}=2.0, \lambda_{1}=1.5, \lambda_{2}=0.5, \lambda_{3}=1.0, \mu_{0}=4.0, \\
\sigma_{0}^{2}=2.0, \mu_{1}=6.0, \sigma_{1}^{2}=1.0
\end{gathered}
$$

We solved the example model on a $933 \mathrm{MhZ}$ Pentium III notebook equipped with $256 \mathrm{MB}$ of RAM running the Windows 2000 operating system and using the cygwin Unix emulator environment. Table 2 presents the CPU times required using the proposed technique, and the direct solution of the discretized system of equations using Gauss-Seidel with sparse matrix implementation (that we refer to as standard technique) for systems with 7 producers and 5 consumers (yielding a model with 48 discrete states) for decreasing values for $\Delta x$, while Table 3 reports CPU times for a system with 20 producers and 10 consumers (yielding a model with 231 discrete states) for increasing values for the buffer size $B_{0}$. Both scenarios aim to evaluate the efficiency of the solution algorithms as the number of discretization points increases. For decreasing values of $\Delta x$ we observe that the CPU time required to solve the model using the new solution technique is almost constant while the CPU times markedly increase when the number of discretization points increases using the standard 
Table 4

CPU times for fixed $B_{0}$ and $\Delta x$ for increasing number of discrete states.

\begin{tabular}{|r|c|c|c|c|c|r|r|}
\hline \hline $\mathrm{N}$ & $\mathrm{K}$ & $|\mathcal{S}|$ & $B_{0}$ & $\Delta X$ & $m+1$ & standard & new \\
\hline \hline 3 & 2 & 12 & 1.0 & 0.1 & 10 & $<1 \mathrm{~s}$. & $<1 \mathrm{~s}$. \\
\hline 5 & 2 & 18 & 1.0 & 0.1 & 10 & $<1 \mathrm{~s}$. & $<1 \mathrm{~s}$. \\
\hline 7 & 2 & 24 & 1.0 & 0.1 & 10 & $<1 \mathrm{~s}$. & $<1 \mathrm{~s}$. \\
\hline 7 & 5 & 48 & 1.0 & 0.1 & 10 & $23 \mathrm{~s}$. & $<1 \mathrm{~s}$. \\
\hline 9 & 5 & 60 & 1.0 & 0.1 & 10 & $40 \mathrm{~s}$. & $2 \mathrm{~s}$. \\
\hline 11 & 7 & 96 & 1.0 & 0.1 & 10 & $141 \mathrm{~s}$. & $5 \mathrm{~s}$. \\
\hline 25 & 15 & 416 & 1.0 & 0.1 & 10 & n.c. & $1,443 \mathrm{~s}$. \\
\hline 40 & 20 & 861 & 10.0 & 0.1 & 100 & n.c. & $8,333 \mathrm{~s}$. \\
\hline \hline
\end{tabular}

solution technique.

In the case of increasing $B_{0}$, the CPU times for the new solution technique are at least one order of magnitude lower than the times required by the standard algorithm. For high values of $B_{0}$ the impact of the matrix multiplication (to be iterated $m+1$ times) increases and becomes the predominant complexity issue making the whole process linearly dependent on $m$.

Furthermore, Table 4 presents the CPU times for the solution of models where both $B_{0}$ and $\Delta x$ are fixed for increasing number of discrete states. In this case, the CPU times for the new solution technique remain at least one order of magnitude lower than the times required by the standard algorithm. Nevertheless, for models with a larger number of discrete states the impact of the matrix inversion required to compute $\Gamma$ and $\Lambda$ increases and becomes the predominant complexity issue making the whole process $O\left(|\mathcal{S}|^{3}\right)$.

\section{An application of second-order FSPN to the analysis of TCP}

In the previous section we proved the effectiveness of our solution technique for second-order FSPN by comparing the CPU times required to solve a complex reference model. In this section we investigate the effectiveness of the proposed solution technique when the model of a complex system is solved according to a decomposition into simpler sub-models (where at least one is a second-order FSPN).

Decomposition is a well-known approach that is employed to analyze complex systems: a model for the whole system is partitioned into several (simpler) 
sub-models and the interaction among the different sub-models is handled by iterating their solution until the complete model solution converges according a fixed point algorithm.

A survey of all previous work based on the decomposition technique is impractical; among all research areas where this approach has been successfully employed one of the most interesting is the analysis of the Transmission Control Protocol (TCP), the connection oriented transport protocol whose congestion control algorithm contributes to dictate the latency of information transfer in the Internet.

TCP has been subject to numerous performance studies based on simulations, measurements, and analytical models. The literature on analytical models of TCP is vast and it is difficult to provide here a comprehensive summary of previous contributions. In this section we only summarize some of the approaches that have been used so far in this field and that are based on a decomposition of a complex model into different sub-models.

Works in $[1,5]$ cope with finite TCP connections; their peculiarity is the analysis of connections that exhibit an on-off behavior, following a Markov model. In these works, the description of the protocol behavior is decoupled from the description of the network behavior and the interaction between the two sub-models is handled by iterating the solution of the sub-models until the complete model solution converges according a fixed point algorithm. In [1] the GSPN formalism was used for the specification of the TCP and the network behavior, while in [5] simple analytical models were developed.

The approach presented in [11] is based on the use of queuing network models for modelling the behavior of the window protocol. These works are based on the description of the protocol through a queuing network model that allows the estimation of the load offered to the IP network (that is also represented by means of a queuing network). The merit of the queuing network approach lies in the product form solution that these models exhibit thus leading to more efficient computation of the interesting performance indices.

The work in [10] addresses the problem of modelling and analyzing the distribution of the completion time as well as the packet loss probability of a set of homogeneous TCP connections that behave as ON-OFF data sources. Their analysis was based on separate Stochastic Petri Nets (SPN) model descriptions for the latency of TCP connections and for the IP network link that are iteratively solved using a fixed point method. In that paper, the IP link was represented by a Generalized Stochastic Petri Nets (GSPN) model. The solution complexity of the GSPN model was highly dependent on the number of ON-OFF sources, the maximum size of the TCP congestion window, and the IP link buffer size. The state-space explosion problem limited the applicability 
and usefulness of the approach since the analysis of large system, e.g., an IP link with a large buffer size, was impractical.

We chose the work in [10] as a test-bed to further prove the effectiveness of our solution technique. We extend and improve that work by developing a second-order FSPN model of the IP link. This is a two-fold contribution:

- a second-order FSPN model of the IP link allows the representation of transmission systems where the buffer size and the maximum size of the TCP congestion window are not a limit;

- this extension represents, to the best of our knowledge, one of the few practical exploitations of the second-order FSPN formalism.

In the following we only summarize the network scenario, the assumptions on the behavior of the TCP connections, as well as the decomposition technique and iterative solution method used to solve the model by the authors of [10]. For details on the models the reader should refer to the original work.

\subsection{The Network Scenario}

A set of K homogeneous TCP Reno connections is considered; each connection is characterized by:

- an ON-OFF behavior where the OFF periods are exponentially distributed with average $T_{\text {silence; }}$

- when in the ON (active) state, a connection transfers $j$ packets with a given probability $\left(g_{j}\right)$, i.e., a TCP source is characterized by a packet emission distribution $g=\left\{g_{j}\right\}$ where $g_{j}=P\{\#$ Packets to transfer $=j\}$;

- same routing in the network;

- similar round trip times (RTT) and packet loss probabilities $(p)$;

- same maximum value for the congestion window $\left(W_{\max }\right)$;

- same fixed packet size (PK bytes).

A simple bottleneck link connecting $K$ TCP senders to their receivers was the considered topology. The link has a capacity of $C \mathrm{Mb} / \mathrm{s}$, propagation delay of $P D \mathrm{~ms}$, and is associated with a drop-tail queue whose size is denoted as $M$. The time to transmit a packet (in seconds) is then given by $\frac{8 * P K}{C}$ (denoted as Transmit). 


\subsection{The Modelling Approach}

The technique proposed in [10] is based on separate descriptions of a TCP source that must transfer $j$ packets, and the IP network links. The TCP source sub-model describes the behavior of one among $K$ connections sharing homogeneous characteristics whose solution yields the average time required to transfer $j$ packets over a route through the network experiencing a given round trip time $R T T$ and a packet loss probability $p$.

The IP network link sub-model is a GSPN description of a finite buffer queue with batch arrivals modulated by an ON-OFF process. A high-level description of the proposed modelling approach (adapted from [10]) is depicted in Figure 4. The TCP source sub-model receives as inputs from the IP network link

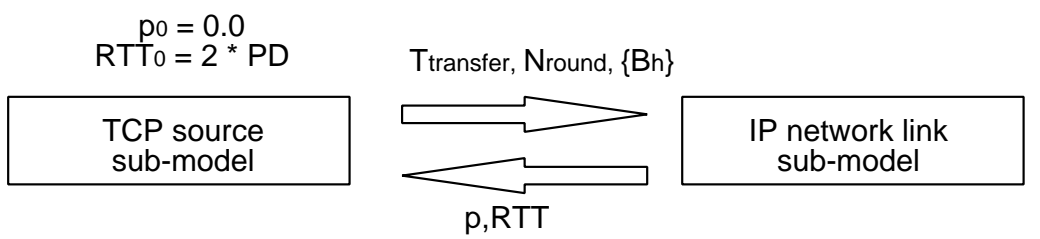

Fig. 4. High level description of the proposed approach

sub-model the following parameters:

- estimate of the packet loss probability;

- estimate of the RTT summing up the average queuing delay for accepted packets, packet transmission time, and constant propagation delays.

The solution of the TCP source sub-model provides an estimate of the average completion time denoted as $T_{\text {transfer }}=E\left\{T_{\text {transfer }}(j)\right\}=\sum_{j} g_{j} *$ $T_{\text {transfer }}(j)$, the average number of rounds ${ }^{1}$ required to complete the packets transfer denoted as $N_{\text {round }}=E\left\{N_{\text {round }}(j)\right\}=\sum_{j} g_{j} * N_{\text {round }}(j)$, the round size distribution denoted as

$$
B_{h}=P\{\text { congestion window size }=h\}=\sum_{j} g_{j} * B_{j, h}
$$

Based on these performance indices, it is also defined $T_{\text {round }}=\frac{{ }^{T} \text { transfer }}{{ }^{N} \text { round }}$ as the average time between successive rounds arrival, and $\bar{B}=\sum_{h=1}^{W \max } h * B_{h}$ as

1 A round begins with the transmission of a window of $W$ packets and ends when the sender receives an acknowledgment for one or more of these packets, e.g., see $[20]$. 
the average round size.

The IP network link sub-model receives as inputs from the TCP source submodel the following parameters:

- estimate of the average completion time for the modulating round arrival process $\left(T_{\text {transfer }}\right)$;

- average number of rounds required to complete the packets transfer $\left(N_{\text {round }}\right)$;

- round size distribution $\left(\left\{B_{h}\right\}\right)$.

The estimates produced by the IP network link sub-model are used as input parameters to the TCP source sub-model in an iterative process until convergence is reached.

\subsection{The second-order FSPN sub-model of the IP network link}

The new model we developed to represent an IP network link traversed by $K$ ON-OFF TCP connections is depicted in Figure 5. The finite capacity buffer of

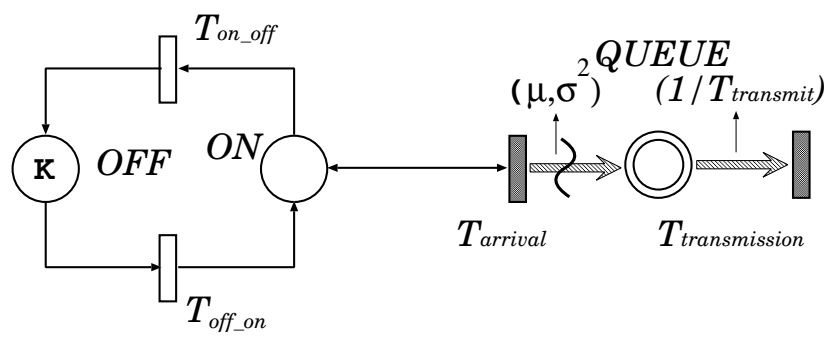

Fig. 5. Second-order FSPN model of the IP network link

the IP link is represented by the fluid place QUEUE. The link transmission rate is represented by the fluid transition $T_{\text {transmission; }}$, the fluid arc connecting fluid place QUEUE to fluid transition $T_{\text {transmission }}$ has constant rate equal to the IP link capacity.

The queue arrival process is the superposition of $K$ homogeneous, two state Markov Modulated Poisson process with fluid arrivals; the mean time spent in the OFF state is one of the network parameters: the OFF periods are exponentially distributed with average $T_{\text {silence }}$ and transition $T_{\text {off_on }}$ models the awakening of a TCP source. The mean time spent in the ON state is input from the TCP latency model and is given by $T_{\text {transfer }}$ transition $T_{\text {on_off }}$ models the connection termination.

In Figure 6 a sample of the packet arrival from one TCP source is depicted. The mean time between round arrivals is given by $T_{\text {round }}$ and the round size distribution is given by $\left\{B_{h}\right\}$. This implies that the average round duration is 
given by $\bar{B} \cdot T_{\text {transmit }}$; the probability of packets arrivals in an activity period can then be defined as

$$
p_{\text {active }}=\frac{\bar{B} \cdot T_{\text {transmit }} \cdot N_{\text {round }}}{T_{\text {transfer }}}
$$

. We then set the mean and variance of the fluid arc connecting place ON to

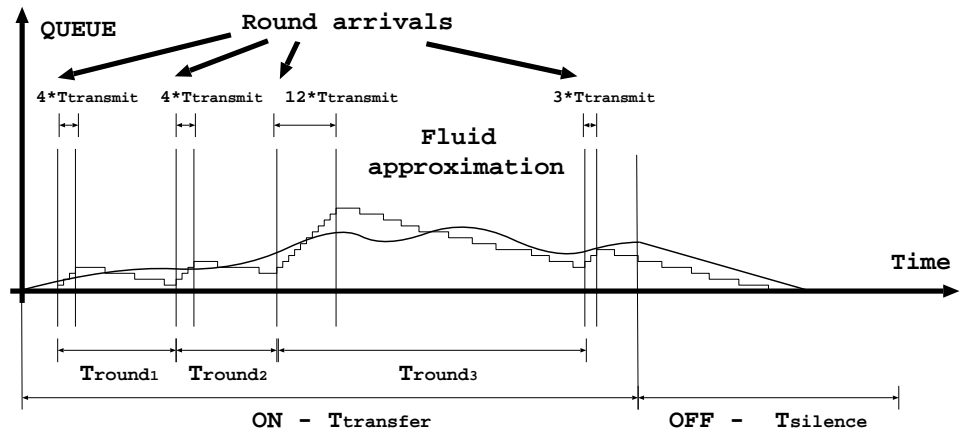

Fig. 6. Arrival pattern sample for one ON-OFF TCP source.

fluid transition $T_{\text {arrival }}$ : the mean flow rate of the second-order fluid (denoted as $\mu$ ) representing the packet arrival is set equal to

$$
\mu=\left(1-p_{\text {active }}\right) \cdot 0+\sum_{h=1}^{W \max } B_{h} \cdot p_{\text {active }} \frac{h}{T_{\text {transmit }} \cdot \bar{B}}=\frac{p_{\text {active }}}{T_{\text {transmit }}}
$$

that is, the rate of packet transmission of the IP link, multiplied by the probability of packet arrivals in the ON periods. The variance of the fluid flow (denoted as $\sigma^{2}$ ) is then defined as

$$
\sigma^{2}=\left(1-p_{\text {active }}\right) \cdot(0-\mu)^{2}+\sum_{h=1}^{W_{\max }} B_{h} \cdot p_{\text {active }}\left(\frac{h}{T_{\text {transmit }} \cdot \bar{B}}-\mu\right)^{2}
$$

When a connection awakes, i.e., transition $T_{\text {off_on }}$ fires, fluid starts to flow in place QUEUE following a fluid process with mean equal to $\mu$ and variance equal to $\sigma^{2}$.

Since the buffer occupancy is approximated by a continuous variable, the probability of having exactly $n$ packets in the buffer, is computed by integrating the probability density of the fluid level [8]: $p_{i}(n)=\int_{n-\frac{1}{2}}^{n+\frac{1}{2}} p_{i}(x) d x$ where $p_{i}(n)$ corresponds to the probability of having $n$ packets in the buffer in discrete state $i$, and $p_{i}(x)$ is probability density of having a fluid level of $x$ in state $i$. In particular: $p_{i}(0)=\int_{0}^{\frac{1}{2}} p_{i}(x) d x$ and $p_{i}(M)=\int_{M-\frac{1}{2}}^{M} p_{i}(x) d x$ which are used respectively to compute the system utilization and the packet loss probability. If we choose the discretization step $\Delta x=\frac{1}{2}$, then $p_{i}(0)$ simply becomes the first discretization point and $p_{i}(M)$ the last discretization point. 
The mean fluid level in place QUEUE is then computed as: $\overline{Q U E U E}=$ $\sum_{i} \int_{0}^{M} x \cdot p_{i}(x) d x$ while the packet loss probability and the round trip time

(defined as $R T T=2 \cdot P D \cdot \frac{\overline{Q U E U E}}{C}$ ), are then used as input parameters to the TCP connection latency model until convergence is reached.

\subsection{Model validation}

This section provides a validation of the new second-order FSPN model for the IP link; we specified the models in [10] and conducted a comparisons of our model's results with the output of the simulator ns-2 [19].

We considered the same validation scenarios presented in [10] and used the same simulation parameters: all simulations were run using a batch method, with a confidence level equal to $97.5 \%$, and accuracy equal to $10 \%$ for the interval estimation of the packet loss probability. An initial transient equal to 300 seconds of simulated time was discarded for each run. Batch width was uniformly distributed between 50 and 70 seconds of simulated time. In all the graphs shown in this section the average packet loss probabilities are plotted using a logarithmic scale for the $y$-axis.

All scenarios share the following characteristics: maximum congestion window size $W_{\max }$ equal to 21 , drop-tail buffer whose size $M$ is equal to 32 packets, and one-way link propagation delay $P D$ equal to $10 \mathrm{~ms}$. As in [10], the TCP source sub-model has been solved using the TimeNET software tool [27], while the IP network link sub-model has been solved using second-order FSPN solution software we developed.

In [10] four validation scenarios were considered whose descriptions are summarized in Table 5. Two packet emission probability distributions have been considered: $g^{\prime}=\left\{g_{1}, g_{3}, g_{5}, g_{8}, g_{12}, g_{19}, g_{35}, g_{52}\right\}$ where $g_{1}=0.3, g_{3}=g_{5}=g_{8}=$ $g_{12}=g_{19}=g_{35}=g_{52}=0.1$, and $g^{\prime \prime}=\left\{g_{1}, g_{8}, g_{35}, g_{52}\right\}$ where $g_{1}=g_{8}=0.3$, $g_{35}=g_{52}=0.2$ for both 30 and 50 TCP connections. In Figure 7 the average packet loss probabilities for all four scenarios are plotted versus increasing values of the average OFF period $T_{\text {off }}$. It can be seen that the model provides an extremely accurate representation of this performance metric both for 30 and for 50 TCP connections. Due to space limitations, results for the Cumulative Distribution Function and for the average completion time are not shown; nevertheless, it must be pointed out that similar accurate results were obtained for these performance indices for all the considered scenarios. 
Table 5

Validation scenarios characterization.

\begin{tabular}{|l|r|r|c|}
\hline \hline & Link capacity & Packet size & Distribution \\
\hline \hline Scenario 1 & $45 \mathrm{Mb} / \mathrm{s}$ & $512 \mathrm{~B}$ & $g^{\prime}$ \\
\hline Scenario 2 & $45 \mathrm{Mb} / \mathrm{s}$ & $512 \mathrm{~B}$ & $g^{\prime \prime}$ \\
\hline Scenario 3 & $100 \mathrm{Mb} / \mathrm{s}$ & $1024 \mathrm{~B}$ & $g^{\prime}$ \\
\hline Scenario 4 & $100 \mathrm{Mb} / \mathrm{s}$ & $1024 \mathrm{~B}$ & $g^{\prime \prime}$ \\
\hline \hline
\end{tabular}
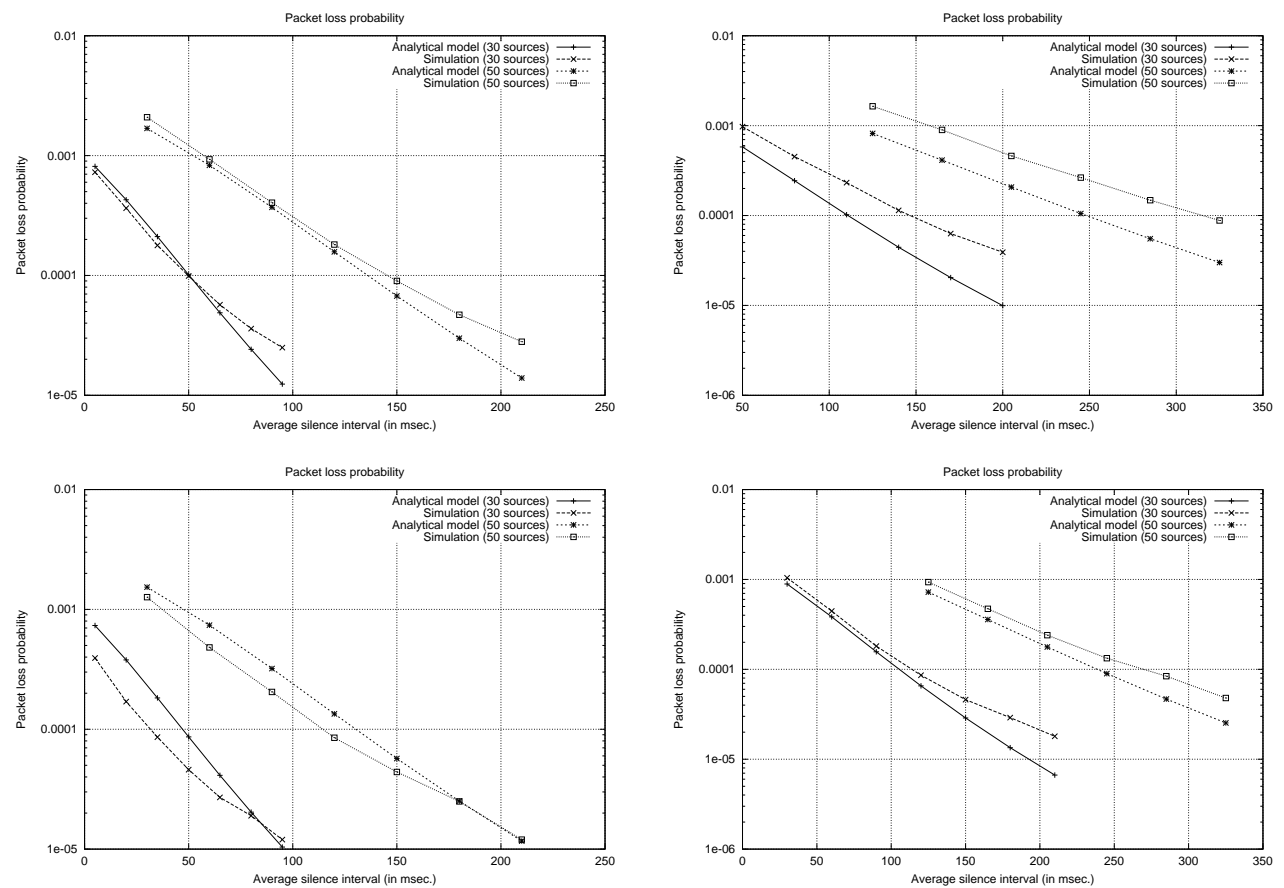

Fig. 7. Average packet loss probability versus average OFF periods for Scenario 1 (upper left), Scenario 2 (upper right), Scenario 3 (lower left), and Scenario 4 (lower right).

\subsection{Model solution complexity}

The number of iterations before convergence is reached depends on the accuracy imposed between successive computations of the average packet loss probability; we used the same stopping criterion defined in [10], i.e., when the relative error $\left(\left|\frac{\text { ploss }_{n+1}-\text { ploss }}{n}\right|\right)$ becomes less than $10^{-2}$ (ploss $s_{n}$ denotes the packet loss probability computed at the $\mathrm{n}^{\text {th }}$ step). The number of iterations in the settings we considered are small, ranging from 2 to 14 for one point of a curve. This range should be compared with the maximum number of iterations for one point of a curve reported in [10] that was equal to 26.

The total CPU time for obtaining the model curves presented in this paper 
was 6,988 seconds on a computer with the same technical specification of [10]. This CPU time should be compared to 12,995 seconds as reported in [10]. ${ }^{2}$ This implies that the new second-order FSPN model and the solution technique we developed almost halved the total CPU time required to obtain all the model results without lowering their accuracy. Furthermore, it should also be noted that the complexity of the solution for the second-order FSPN model is only dependent on the number of discrete states that, in our case, is equal to $K+1$ where $K$ is the number of TCP connections.

\section{Conclusions}

The paper presented an efficient solution technique for the steady-state analysis of the second-order stochastic fluid model underlying a second-order FSPN with constant flow and transition rates and variance, and a single fluid place. The new solution technique is an extension of existing solution techniques developed for first-order fluid models and uses upwind semi-discretization and Matrix Geometric techniques to efficiently compute the steady-state joint probability of the discrete states and the fluid level.

We proved the effectiveness of our technique on a simple producer-consumer second-order FSPN model; furthermore, we developed a second-order FSPN model for a complex example taken from the literature where the analysis of the completion time distribution and the packet loss probability of short-lived TCP connections. The investigation is carried out through the decomposition of a SPN model for the whole system into several (simpler) sub-models; the interaction among the different sub-models is handled by iterating their solution until the complete model solution converges according a fixed point algorithm. The introduction of a second-order FSPN in a fixed point iteration scheme, has been made feasible thanks to the efficiency of the proposed solution technique.

Future development of this work can be sought: first of all, generalization of our solution technique for second-order FSPN with fluid-dependent flow rates is currently under investigation.

As for the application of second-order FSPN models to the analysis of shortlived TCP connections, we are currently working to the characterization of the output process of the second-order FSPN model of the IP network link; the goal is to decompose a multi-link scenario (a network of multiple routers) loaded with short-lived TCP traffic into separate second-order FSPN models

2 In both cases, the CPU time for the models solution must be compared with several days of CPU times for the $n s$ simulator to produce point estimates. 
of each node traversed by TCP traffic.

\section{References}

[1] M. Ajmone Marsan, C. Casetti, R. Gaeta, and M. Meo. Performance analysis of TCP connections sharing a congested internet link. Performance Evaluation, 42(2-3), September 2000.

[2] H. Alla and R. David. Continuous and Hybrid Petri Nets. Journal of Systems Circuits and Computers, 8(1), Feb 1998.

[3] E. Ang and J. Barria. The Markov modulated regulated brownian motion: a second-order fluid flow model of a finite buffer. Queueing Systems, 35:263-287, 2000 .

[4] D. Anick, D. Mitra, and M. M. Sondhi. Stochastic Theory of a Data-Handling System. Bell Sys. Thech. J, 61(8):1871-1894, Oct 1982.

[5] C. Casetti and M. Meo. A New approach to Model the Stationary Behavior of TCP Connections. In Proc. IEEE Infocom 2000, Tel Aviv, Israel, March 2000. IEEE Comp. Soc. Press.

[6] D. Chen, Y. Hong, and K. Trivedi. Second-order stochastic fluid models with fluid-dependent flow rates. Performance Evaluation, 49(1-4):341-358, 2002.

[7] G. Ciardo, D. M. Nicol, and K. S. Trivedi. Discrete-event Simulation of Fluid Stochastic Petri Nets. IEEE Transactions on Software Engineering, 2(25):207217, 1999.

[8] T. Czachórski and F. Pekergin. Diffusion approximation as a modelling tool. In Tutorial Proceedings of the $8^{\text {th }}$ IFIP Workshop on Performance Modelling and Evaluation of ATM $\&$ IP Networks, Ilkley, U.K., July 2000.

[9] A. I. Elwalid and D. Mitra. Statistical Multiplexing with Loss Priorities in Rate-Based Congestion Control of High-Speed Networks. IEEE Transaction on Communications, 42(11):2989-3002, November 1994.

[10] R. Gaeta, M. Manini, and M. Sereno. Stochastic Petri Nets Models for the Performance Analysis of TCP Connections Supporting Finite Data Transfer. In Proceeding of Second International Workshop on Quality of Service in Multiservice IP Networks 2003, QoS-IP 2003, LNCS, N. 2601, pages 372-391, Milano, Italy, Feb 2003. Springer Verlag.

[11] M. Garetto, R. Lo Cigno, M. Meo, and M. Ajmone Marsan. A Detailed and Accurate Closed Queueing Network Model of Many Interacting TCP Flows. In Proc. IEEE Infocom 2001, Anchorage, Alaska, USA, 2001. IEEE Comp. Soc. Press. 
[12] R. German, M. Gribaudo, G. Horvath, and M. Telek. Stationary analysis of FSPNs with mutually dependent discrete and continuous parts. In Proc. 10th Int. Workshop on Petri Nets and Performance Models (PNPM'03), pages 3039, Urbana-Champaign, USA, Sept. 2003. IEEE Comp. Soc. Press.

[13] M. Gribaudo and A. Horvath. Fluid stochastic Petri nets augmented with flush-out arcs: A transient analysis technique. IEEE Transactions on Software Engineering, 28:944-955, Oct. 2002.

[14] M. Gribaudo, M. Sereno, A. Bobbio, and A. Horvath. Fluid Stochastic Petri Nets augmented with Flush-out arcs: Modelling and Analysis. Discrete Event Dynamic Systems, 11(1 \& 2), 2001.

[15] G. Horton, V. G. Kulkarni, D. M. Nicol, and K. S. Trivedi. Fluid stochastic Petri Nets: Theory, Application, and Solution Techniques. European Journal of Operations Research, 105(1):184-201, Feb 1998.

[16] A. Horváth and M. Gribaudo. Matrix geometric solution of fluid stochastic Petri nets. In Proc. of 4th International Conference on Matrix-Analytic Methods in Stochastic models, Adelaide, Australia, July 2002.

[17] R. J. Karandikar and V. G. Kulkarni. Second-Order Fluid Flow Models: Reflected Brownian Motion in a Random Environment. Operations Research, 43(1):77-88, 1995.

[18] G. Latouche and V. Ramaswami. Introduction to Matrix Geometric Methods in Stochastic Modeling. ASA-SIAM Series on Statistics and Applied Probability. SIAM, Philadelphia PA, 1999.

[19] S. McCanne and S. Floyd. ns-2 network simulator (ver.2). Technical report, 1997. URL http://www.isi.edu/nsnam/ns/.

[20] J. Padhye, V. Firoiu, D. Towsley, and J. Kurose. Modeling TCP Reno performance: a simple model and its empirical validation. IEEE/ACM Transaction on Networking, 8(2):133-145, 2000.

[21] B. Sericola. Transient analysis of stochastic fluid models. Performance Evaluation, 1(32):245-263, May 1998.

[22] K. Trivedi and V. Kulkarni. FSPNs: Fluid Stochastic Petri nets. In Application and Theory of Petri Nets 1993, Proc. $14^{\text {th }}$ Intern. Conference, LNCS, Chicago, USA, June 1993. Springer Verlag.

[23] K. Wolter. Second Order Fluid Stochastic Petri Nets: an Extension of GSPNs for Approximate and Continuous Modelling. In Proc. of World Congress on System Simulation, pages 328-332, Singapore, Sep 1997.

[24] K. Wolter. Jump Transitions in Second Order FSPNs. In Proc. of MASCOTS'99, Washington, DC, Oct 1999.

[25] K. Wolter, G. Horton, and R. German. Non-Markovian Fluid Stochastic Petri Nets. Technical report, Tecnical University of Berlin, Berlin, Germany, 1996. Report 1996-13. 
[26] K. Wolter, A. Zisowsky, and G. Hommel. Performance models for a hybrid reactor system. In Modelling, Analysis, and Design of Hybrid Systems, Lecture Notes on Control and Information Sciences, N. 279, pages 193-210. Springer Verlag, 2002.

[27] A. Zimmermann, R. German, J. Freiheit, and G. Hommel. TimeNET 3.0 Tool Description. In $8^{\text {th }}$ Intern. Workshop on Petri Nets and Performance Models, Zaragoza, Spain, Sep 1999. IEEE-CS Press. 